\title{
BMJ Open Catheter-related infections: does the spectrum of microbial causes change over time? A nationwide surveillance study
}

Niccolò Buetti, ${ }^{1}$ Elia Lo Priore, ${ }^{1}$ Andrew Atkinson, ${ }^{1}$ Andreas F Widmer, ${ }^{2}$ Andreas Kronenberg, ${ }^{3}$ Jonas Marschall, ${ }^{1}$ the Swiss Centre for Antibiotic Resistance (ANRESIS)

To cite: Buetti N, Lo Priore E, Atkinson A, et al. Catheterrelated infections: does the spectrum of microbial causes change over time? A nationwide surveillance study. BMJ Open 2018;8:e023824. doi:10.1136/ bmjopen-2018-023824

- Prepublication history and additional material for this paper are available online. To view these files, please visit the journal online (http://dx.doi org/10.1136/bmjopen-2018023824).

$\mathrm{NB}$ and ELP contributed equally.

Received 1 May 2018

Revised 27 September 2018

Accepted 14 November 2018

Check for updates

(c) Author(s) (or their employer(s)) 2018. Re-use permitted under CC BY-NC. No commercial re-use. See rights and permissions. Published by BMJ.

${ }^{1}$ Department of Infectious Diseases, University Hospital Bern, Bern, Switzerland ${ }^{2}$ Division of Infectious Diseases and Hospital Epidemiology, University Hospital Basel, Basel, Switzerland

${ }^{3}$ Institute for Infectious Diseases, University of Bern, Bern,

Switzerland

Correspondence to

Dr Niccolò Buetti;

niccolo.buetti@gmail.com

\section{ABSTRACT}

Objectives To estimate the incidence and epidemiology of catheter-related bloodstream infections (CRBSIs) on a national scale by using prospective epidemiological data from the Swiss Antibiotic Resistance Surveillance System (ANRESIS).

Design Observational study.

Setting National surveillance from 2008 to 2015 of acute hospitals in Switzerland.

Participants We included acute Swiss hospitals that sent blood cultures and catheter tip culture results on a regular basis during the entire study period to the ANRESIS database.

Outcome measure A catheter-related bloodstream infection (termed 'modified CRBSI', mCRBSI) was defined as isolating the same microorganism with identical antibiogram from $\geq 1$ blood cultures (performed \pm 7 days around the catheter removal) as the one recovered from the catheter tip. Incidence rates of mCRBSI were calculated per 1000 admissions.

Results From 2008 to 2015, the mCRBSI incidence rate decreased from 0.83 to 0.58 episodes/1000 admissions $(-6 \%$ per year, $\mathrm{p}<0.001)$. Coagulase-negative staphylococci, Staphylococcus aureus and fungi all exhibited decreasing trends, while rates of enterococci and Gram-negative bacteria remained stable.

Conclusions The overall incidence of mCRBSI in Switzerland is decreasing; however, the incidence of mCRBSI due to Enterococci and Gram-negative microorganisms did not change over time. These pathogens may grow in importance in catheter-related infections, which would have clinical implications for the choice of empirical treatment.

\section{INTRODUCTION}

Catheter-related bloodstream infection (CRBSI) or central line-associated bloodstream infections (CLABSIs) are associated with increased morbidity, mortality and healthcare costs. ${ }^{1}$ The epidemiology of CLABSI has occasionally been evaluated on a national scale; however, studies focused for the most part on the intensive care unit
Strengths and limitations of this study

- Provides an estimation of catheter-related bloodstream infection (CRBSI) on a national scale, an aspect rarely investigated in European countries.

- The observed trends should not have been affected by selection bias given the use of the same CRBSI definition throughout the study period.

- While surveillance studies on central line-associated bloodstream infection or CRBSI mostly focused on overall incidence rates, we also determined the pathogen distribution.

- No clinical data were available (patient and catheter data).

- Only those CRBSI episodes for which the catheter was removed and submitted to the laboratory were included.

(ICU) setting. ${ }^{2}{ }^{3}$ In contrast, very few studies investigated CLABSI outside the ICU. ${ }^{4-7}$ Of note, the term CLABSI is used for surveillance purposes (where the definition neither requires quantitative criteria nor a microbiological diagnosis of the removed catheter tip), whereas the source of infection in CRBSI is based on a positive culture of the catheter tip. CLABSI surveillance can therefore easily lead to an overestimation of the incidence of CRBSI. ${ }^{8}$ More specific definitions based on single-institution surveillance studies have previously been proposed, including the use of admissions ${ }^{9}{ }^{10}$ or bed-days ${ }^{11}$ as denominator. Such a definition permits the identification of the catheter as source of infection, considering both catheter tip culture results and blood cultures (ie, a 'modified' CRBSI). Moreover, the incidence of CRBSI has rarely been investigated on a national scale in European countries, given the difficulty in obtaining clinical information. Here, we wanted to perform a first estimation of 
changes in the epidemiology of CRBSI in Switzerland according to the 'modified' CRBSI definition, using a national microbiological surveillance database.

\section{METHODS}

We conducted a nationwide, observational study on CRBSI using Swiss Antibiotic Resistance Surveillance System (ANRESIS) data from 2008 to 2015. The ANRESIS programme summarises all positive blood and catheter tip cultures from 20 Swiss laboratories, each of them collecting data from several hospitals distributed across the country. Accordingly, we analysed data of patients from 36 Swiss hospitals, including only those centres that sent catheter tip information on a regular basis during the entire study period. All five Swiss university hospitals and the main regional hospitals were included, representing the majority of hospitalised patients in the country during the study period.

A catheter tip was included in the analysis if at least one microorganism could be cultivated, irrespective of the cut-off of the roll plate method. ${ }^{12}$ In case of a polymicrobial CBRSI, each microorganism isolated was considered as a single event. Information about the microbiological method (quantitative sonication vs semiquantitative roll plate culture) was not routinely made available by the participating laboratories. However, in a previous analysis using a similar dataset, $83 \%$ of the participating laboratories used the semiquantitive roll plate culture method. ${ }^{13}$ Additional culture tip reports of another catheter tip with the same microorganism in the same patient within 7 days were excluded.

A catheter-related bloodstream infection, here termed 'modified CRBSI' (mCRBSI), was defined as isolating the same micro-organism with identical antibiogram from $\geq 1$ blood cultures (performed \pm 7 days around the catheter removal) as the one recovered from the catheter tip. CRBSI episodes diagnosed by differential time-to-positivity or quantitative blood cultures could not be included because this information was not available from the participating laboratories. Incidence rates of mCRBSI were calculated per 1000 admissions using national data on hospital statistics. ${ }^{14}$ With this definition, a satisfactory correlation between mCRBSI and CLABSI was previously documented. ${ }^{9}$ From 2008 to 2015, an increase of hospital admissions was observed ${ }^{14}$; therefore, a supplementary analysis using hospital-days as denominator was performed. ${ }^{14}$ Moreover, a supplementary analysis using a stricter definition of mCRBSI (isolation of the same microorganism in blood cultures performed -7 days to +2 days around the catheter removal and in the catheter tip cultures) was performed. We then performed trend analyses for the following micro-organism groups: Staphylococcus aureus, coagulase-negative staphylococci (CoNS), enterococci, Enterobacteriaceae, Gram-negative non-fermenters, anaerobes and fungi. For Enterobacteriaceae, Gram-negative non-fermenters and enterococci, we performed a trend analysis of resistance to ceftriaxone, cefepime and vancomycin, respectively. A subanalysis of the following categories was conducted: age $(<65$ vs $\geq 65$ years), gender (male vs female), department (ICU vs non-ICU) and type of hospital (community vs university hospital).

Group comparisons were performed using Student's t-test for normally distributed continuous variables, with the Mann-Whitney-Wilcoxon test for non-normally distributed continuous variables or with Pearson's $\chi^{2}$ test for dichotomous variables. Models for the overall rate increase per year, adjusted for each of gender, age, type of hospital, department and pathogen (seven groups) were fitted in turn using a Poisson regression model including an offset for the estimated admissions/bed-days in the respective year.

Since the analysis was performed from anonymised non-genetic surveillance data, neither approval from an ethics committee nor patient consent was required according to the Swiss law for research on human beings (Art. 33 al. 2 LRH).

\section{Patient and public involvement}

No patients were involved in the design, recruitment or conduct of this study.

\section{RESULTS}

A total of 2741 mCRBSI episodes were reported between 2008 and 2015, with a mean incidence rate of 342 episodes per year. Twenty-six per cent of the episodes $(n=714)$ occurred in ICU departments and 43\% ( $n=1177)$ were detected in university hospitals.

The mCRBSI incidence rate decreased from 0.83 to 0.58 episodes $/ 1000$ admissions during the study period ( $-6 \%$ per year, $\mathrm{p}<0.001$; figure 1$)$. The total number of admissions increased from 469816 in 2008 to 533017 in 2015. A supplementary analysis using patient-days (which remained constant during the study period) as denominator showed similar trends (cf. online supplementary

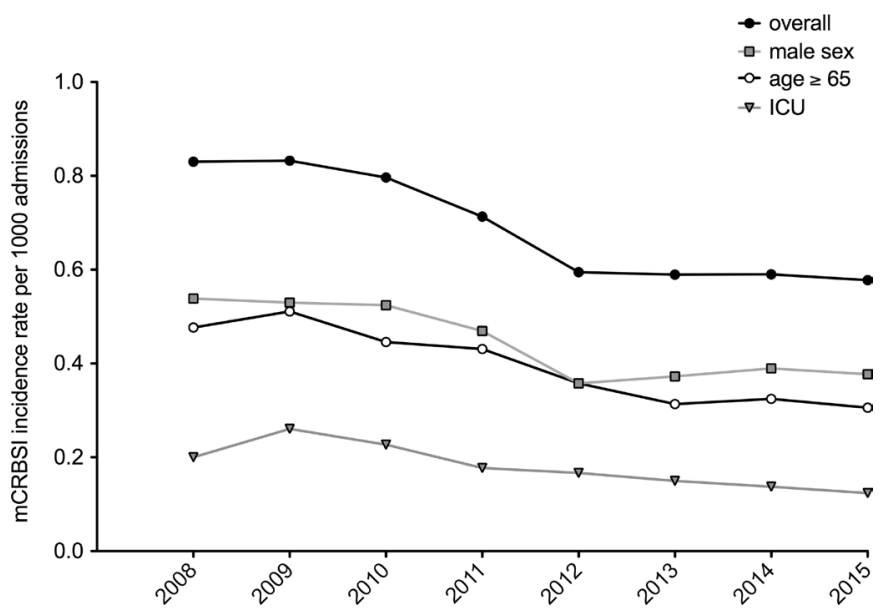

Figure 1 Incidence of modified catheter-related bloodstream infection (mCRBSI) per 1000 hospital admissions: overall trends and subgroups. All trends were significant. ICU, intensive care unit. 


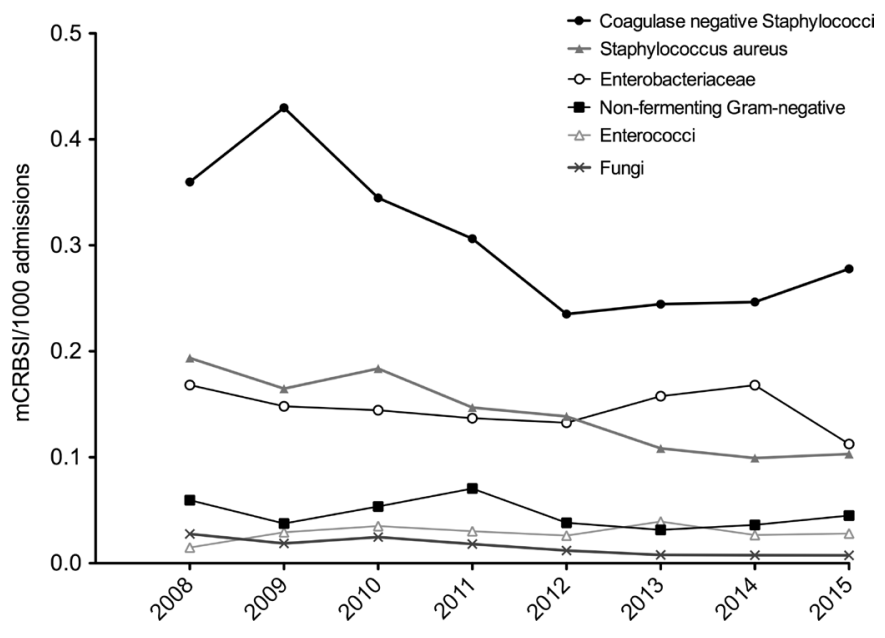

Figure 2 Incidence of mCRBSI caused by CoNS, Staphylococcus aureus, enterococci, Enterobacteriaceae, Gram-negative non-fermenters and fungi. CoNS, coagulasenegative staphylococci; ICU, intensive care unit; mCRBSI, modified catheter-related bloodstream infection.

figures A,B). Moreover, comparable trends were observed when limiting the analysis to bloodstream infection episodes -7 days to +2 days around the catheter removal (cf. online supplementary figure $\mathrm{G}$ ). The most notable trends were observed in individuals aged $\geq 65$ years $(-3 \%$ per year, $\mathrm{p}=0.04)$, in university hospitals $(-4 \%$ per year, $\mathrm{p}=0.009)$ and in ICU departments $(-4 \%$ per year, $\mathrm{p}=0.04)$.

CoNS ( $-7 \%$ per year, $\mathrm{p}<0.001), S$. aureus $(-10 \%$ per year, $\mathrm{p}<0.001)$ and fungi $(-20 \%$ per year, $\mathrm{p}<0.001)$ all exhibited decreasing trends (figure 2 ). On the other hand, enterococci $(+3 \%, \mathrm{p}=0.39)$, Enterobacteriaceae $(-2 \%$ per year, $\mathrm{p}=0.25)$ and Gram-negative non-fermenters $(-5 \%, \mathrm{p}=0.1)$ remained stable over the study period without statistically significant trends. No pathogen group showed an upward rate trend over the study period.

Among Enterobacteriaceae $(n=681)$, a slight non-significant increase in ceftriaxone resistance was observed during the study period. A significant upward trend in vancomycin resistance was noted in enterococci $(n=116)$. No significant trend was found in cefepime resistance in Gram-negative non-fermenters $(n=182)$ (cf. online supplementary figures D1-D3).

\section{DISCUSSION}

Catheter-related infections can either be identified for surveillance purpose (CLABSI) or in clinical terms (CRBSI). Here, we present data from a large surveillance study in 36 hospitals across Switzerland, corresponding to approximately $38 \%$ of all national hospital admissions in $2015^{14}$. Our study appears to mirror a trend of decreasing incidence of CLABSI seen elsewhere, using the more precise and clinically oriented definition of mCRBSI. Our report could be of particular interest to other countries where no nationwide surveillance of CLABSI has been established yet. Indeed, the main finding of this analysis is that, overall, mCRBSI decreased both in ICU and
non-ICU patients. A comparison with other surveillance studies is difficult, since few studies relied on this (or a similar) case definition. ${ }^{101115}$ Our definition was in fact closely linked to one used by Rodriguez-Créixems et al. ${ }^{9}$ Possible reasons for the observed decrease of the mCRBSI rate may include: (1) implementation of national initiatives aimed at improving standards in hospital infection prevention, ${ }^{16}$ (2) a decreasing average duration of hospitalisation with early discharge of patients potentially prone to develop $\mathrm{CRBSI}^{17}$; (3) changing policies in recommending tip cultures to be taken if a catheter is removed; possibly, catheter tip cultures were less and less frequently recommended by local clinicians over the course of the study period and (4) an increasing number of admissions from 2008 to 2015 could have led to an apparent decrease in the incidence of mCRBSI. However, a supplementary analysis using patient-days as denominator revealed similar trends as the main analysis.

Interestingly, the incidence of CoNS, $S$. aureus and fungi decreased significantly over time, while Gram-negative microorganisms and Enterococci remained stable over the study period. These stable trends are of particular concern, since both Enterococci and Gram-negative infections may be associated with high resistance and mortality rates. ${ }^{18} 19$ A rise in vancomycin resistance in Enterococci was noted, but given that only four samples showed vancomycin resistance this finding remains difficult to interpret. No significant increase in resistance Gram-negative bacteria could be detected. While surveillance studies on CLABSI or CRBSI mostly focused on overall incidence rates, comparatively little attention has been drawn to the pathogen distribution of catheter-related infections. Recently published data showed that enterococcal catheter infections either predominated in terms of pathogen distribution ${ }^{6} 20$ or showed increasing trends in two reports. ${ }^{2} 21$ Similar trends or patterns were observed for Gram-negative CRBSI. ${ }^{112223}$ It is conceivable that the improved standards in hospital infection prevention have had less impact on these particular microorganism groups. ${ }^{22}$ An increase in multidrug-resistant strains or the rise in the medical complexity of hospital patients might be further reasons for these trends. ${ }^{23}$ Most epidemiological studies so far have neglected to focus on Gram-negative bacteria and Enterococci as causes of catheter infections. We are convinced that further research should focus on these two subsets of CRBSI.

Our study has several limitations. First, our definition of mCRBSI is highly specific and some cases of catheter-related infections might have been missed. In particular, only those CRBSI in which the catheter was removed and submitted to the laboratory were included, which may have led to an underestimation of the total burden of catheter-related infections. However, the same criteria for identifying CRBSI were used throughout the study period and, therefore, the observed trends should have not be affected. The 
inclusion of cases with only one positive blood culture for commensals could have led to an overestimation of the CRBSI rate (eg, in case of colonisation or contamination). However, by requiring a positive catheter tip we think that this effect has been mitigated. By including episodes up to 7 days after the catheter removal, non-catheter-related infections may have been included. However, a satisfactory correlation between mCRBSI and CLABSI has previously been documented in ICU patients. ${ }^{9}$ Second, neither information on the catheter type nor pertinent clinical data was available, which may have led to the inclusion of peripheral venous/arterial catheters or catheter tips sent without clinical indication. Finally, using admission as denominator, possible changes in device days were not considered. However, we believe that this drawback is outweighed by the advantage of obtaining reliable mCRBSI trends where clinical surveillance was not feasible. We were unable to correlate the CLABSI rates with our results of mCRBSI, since there is no national CLABSI surveillance in Switzerland yet; to assess the gap between rates of CLABSI and mCRBSI on a national scale would be an interesting next step.

Our data suggest that the overall incidence of mCRBSI in Switzerland is decreasing; however, mCRBSI due to Enterococci and Gram-negative microorganisms did not change over time. These pathogens may grow in importance in catheter-related infections, which would have clinical implications for the choice of empirical treatment.

Acknowledgements We thank all microbiology laboratories participating in the Swiss Centre for Antibiotic Resistance (ANRESIS) network: Institute for Laboratory Medicine, Cantonal Hospital Aarau; Central Laboratory, Microbiology Section, Cantonal Hospital Baden; Clinical Microbiology, University Hospital Basel; Viollier AG, Basel; Laboratory Medicine EOLAB, Department of Microbiology, Bellinzona; Institute for Infectious Diseases, University Bern; Microbiology Laboratory, Unilabs, Coppet ; Central Laboratory, Cantonal Hospital Graubünden; Microbiology Laboratory, Hospital Thurgau; Microbiology Laboratory Hôpital Fribourgeois, Fribourg; Bacteriology Laboratory, Geneva University Hospitals, Geneva; ADMED Microbiology, La Chaux-de-Fonds; Institute for Microbiology, Université de Lausanne; Centre for Laboratory Medicine, Cantonal Hospital Luzern; Centre for Laboratory Medicine, Cantonal Hospital Schaffhausen; Centre for Laboratory Medicine Dr. Risch, Schaan; Central Institute, Hôpitaux Valaisans (ICHV), Sitten; Centre of Laboratory Medicine St. Gallen; Institute for Medical Microbiology, University Hospital Zürich; Laboratory for Infectious Diseases, University Children's Hospital Zürich. In addition, we appreciate the steering committee of ANRESIS for supporting this analysis.

Collaborators Swiss Centre for Antibiotic Resistance (ANRESIS): A Burnens, Synlab Suisse, Switzerland; A Cherkaoui, Bacteriology Laboratory, Geneva University Hospitals, Switzerland; V Gaia, Department of Microbiology, EOLAB, Bellinzona, Switzerland; 0 Dubuis, Viollier AG, Basel, Switzerland; A Egli, Clinical Microbiology Laboratory, University Hospital Basel, Switzerland; D Koch, Federal Office of Public Health, Bern, Switzerland; A Kronenberg, Institute for Infectious Diseases, University of Bern, Switzerland; S Luyet, Swiss Conference of the Cantonal Ministers of Public Health, Switzerland; P Nordmann, Molecular and Medical Microbiology, Department of Medicine, University Fribourg, Switzerland; V Perreten, Institute of Veterinary Bacteriology, University of Bern, Switzerland; J-C Piffaretti, Interlifescience, Massagno, Switzerland; G Prod'hom, Institute of Microbiology, Centre Hospitalier Universitaire Vaudois, Lausanne, Switzerland; J Schrenzel, Bacteriology Laboratory, Geneva University Hospitals, Geneva, Switzerland; S Leib, Institute for Infectious Diseases, University of Bern, Switzerland; G Zanetti, Service of Hospital Preventive Medicine, Centre Hospitalier Universitaire Vaudois, Lausanne, Switzerland; R Zbinden, Institute of Medical Microbiology, University of Zürich, Switzerland.
Contributors NB, JM, ELP conceived and designed the study. NB, AA, ELP analysed the data. NB, JM, AK, ELP and AFW wrote the manuscript. All authors contributed to the discussion and reviewed the manuscript. All authors commented and approved the final version of the paper. The ANRESIS programme summarises all positive blood and catheter tip cultures from 20 Swiss laboratories, each of them collecting data from several hospitals distributed across Switzerland.

Funding The authors have not declared a specific grant for this research from any funding agency in the public, commercial or not-for-profit sectors.

Competing interests None declared.

Patient consent for publication Not required.

Provenance and peer review Not commissioned; externally peer reviewed.

Data sharing statement The datasets generated and/or analysed during the current study are not publicly available but are available from the corresponding author on reasonable request.

Open access This is an open access article distributed in accordance with the Creative Commons Attribution Non Commercial (CC BY-NC 4.0) license, which permits others to distribute, remix, adapt, build upon this work non-commercially, and license their derivative works on different terms, provided the original work is properly cited, appropriate credit is given, any changes made indicated, and the use is non-commercial. See: http://creativecommons.org/licenses/by-nc/4.0/.

\section{REFERENCES}

1. Blot SI, Depuydt P, Annemans L, et al. Clinical and economic outcomes in critically ill patients with nosocomial catheter-related bloodstream infections. Clin Infect Dis 2005;41:1591-8.

2. Choi JY, Kwak YG, Yoo H, et al. Trends in the incidence rate of device-associated infections in intensive care units after the establishment of the Korean Nosocomial Infections Surveillance System. J Hosp Infect 2015;91:28-34.

3. Soe MM, Edwards JR, Sievert DM, et al. Evaluating state-specific antibiotic resistance measures derived from central line-associated bloodstream infections, national healthcare safety network, 2011. Infect Control Hosp Epidemiol 2015;36:54-64.

4. Rhee $Y$, Heung M, Chen B, et al. Central line-associated bloodstream infections in non-ICU inpatient wards: a 2-year analysis. Infect Control Hosp Epidemiol 2015;36:424-30.

5. Marschall J, Leone C, Jones M, et al. Catheter-associated bloodstream infections in general medical patients outside the intensive care unit: a surveillance study. Infect Control Hosp Epidemiol 2007;28:905-9.

6. See I, Freifeld AG, Magill SS. Causative organisms and associated antimicrobial resistance in healthcare-associated, central lineassociated bloodstream infections from oncology settings, 20092012. Clin Infect Dis 2016;62:1203-9.

7. Vonberg RP, Behnke M, Geffers C, et al. Device-associated infection rates for non-intensive care unit patients. Infect Control Hosp Epidemiol 2006;27:357-61.

8. Mermel LA, Allon M, Bouza E, et al. Clinical practice guidelines for the diagnosis and management of intravascular catheter-related infection: 2009 Update by the Infectious Diseases Society of America. Clin Infect Dis 2009;49:1-45.

9. Rodríguez-Créixems M, Muñoz P, Martín-Rabadán P, et al. Evolution and aetiological shift of catheter-related bloodstream infection in a whole institution: the microbiology department may act as a watchtower. Clin Microbiol Infect 2013;19:845-51.

10. Bouza E, Eworo A, Fernández Cruz A, et al. Catheter-related bloodstream infections caused by Gram-negative bacteria. J Hosp Infect 2013;85:316-20.

11. Braun E, Hussein K, Geffen Y, et al. Predominance of Gram-negative bacilli among patients with catheter-related bloodstream infections. Clin Microbiol Infect 2014;20:0627-9.

12. Maki DG, Weise CE, Sarafin HW. A semiquantitative culture method for identifying intravenous-catheter-related infection. N Engl J Med 1977;296:1305-9.

13. Buetti N, Lo Priore E, Atkinson A, et al. Low incidence of subsequent bacteraemia or fungaemia after removal of a colonized intravascular catheter tip. Clin Microbiol Infect 2018;24:548.e1-3.

14. Bundesamt für Gesundheit (BAG), (Federal Office of Public Health),Spitalstatistiken. Hospital statistics. (accessed 21 Jan 2017).

15. Strasheim W, Kock MM, Ueckermann V, et al. Surveillance of catheter-related infections: the supplementary role of the microbiology laboratory. BMC Infect Dis 2015;15:5.

16. Swissnoso. www.swissnoso.ch (accessed 28 Jan 2018). 
17. Scheer B, Perel A, Pfeiffer UJ. Clinical review: complications and risk factors of peripheral arterial catheters used for haemodynamic monitoring in anaesthesia and intensive care medicine. Crit Care 2002;6:199-204.

18. ECDC. Antimicrobial resistance surveillance in Europe 2015, 2017.

19. Pinholt M, Ostergaard C, Arpi M, et al. Incidence, clinical characteristics and 30-day mortality of enterococcal bacteraemia in Denmark 2006-2009: a population-based cohort study. Clin Microbiol Infect 2014;20:145-51.

20. Worth LJ, Spelman T, Bull AL, et al. Central line-associated bloodstream infections in Australian intensive care units: Timetrends in infection rates, etiology, and antimicrobial resistance using a comprehensive Victorian surveillance program, 2009-2013. Am J Infect Control 2015;43:848-52.

21. Reigadas E, Rodríguez-Créixems M, Guembe M, et al. Catheterrelated bloodstream infection caused by Enterococcus spp. Clin Microbiol Infect 2013;19:457-61.

22. Lin KY, Cheng A, Chang YC, et al. Central line-associated bloodstream infections among critically-ill patients in the era of bundle care. J Microbiol Immunol Infect 2017;50:339-48.

23. Marcos $M$, Soriano $A$, Iñurrieta $A$, et al. Changing epidemiology of central venous catheter-related bloodstream infections: increasing prevalence of Gram-negative pathogens. J Antimicrob Chemother 2011;66:2119-25. 